Article

\title{
Increased Azithromycin Susceptibility of Multidrug-Resistant Gram-Negative Bacteria on RPMI-1640 Agar Assessed by Disk Diffusion Testing
}

\author{
Milton Meerwein ${ }^{1,+}{ }^{\circledR}$, Andrea Tarnutzer ${ }^{1,+}$, Michelle Böni ${ }^{1}$, Françoise Van Bambeke ${ }^{2}$, \\ Michael Hombach ${ }^{3, \ddagger}$ and Annelies S. Zinkernagel ${ }^{1, *}$ \\ 1 Department of Infectious Diseases and Hospital Epidemiology, University of Zurich, 8091 Zurich, \\ Switzerland; milton@meerwe.in (M.M.); andrea.tarnutzer@usz.ch (A.T.); michelle.boeni@uzh.ch (M.B.) \\ 2 Pharmacologie cellulaire et moléculaire, Louvain Drug Research Institute, Université catholique de Louvain, \\ B-1200 Brussels, Belgium; francoise.vanbambeke@uclouvain.be \\ 3 Institute of Medical Microbiology, University of Zurich, 8091 Zurich, Switzerland; \\ michael.hombach@roche.com \\ * Correspondence: annelies.zinkernagel@usz.ch \\ + These authors contributed equally to this work. \\ $\ddagger$ Current address: Michael Hombach, Roche Diagnostics International AG, 6343 Rotkreuz, Switzerland.
}

Received: 20 March 2020; Accepted: 26 April 2020; Published: 29 April 2020

\begin{abstract}
Increasing antibiotic resistances and a lack of new antibiotics render the treatment of Gram-negative bacterial infections increasingly difficult. Therefore, additional approaches are being investigated. Macrolides are not routinely used against Gram-negative bacteria due to lack of evidence of in vitro effectiveness. However, it has been shown that Pseudomonas spp. are susceptible to macrolides in liquid RPMI-1640 and clinical data suggest improvement in patients' outcomes. So far, these findings have been hardly applicable to the clinical setting due to lack of routine low-complexity antimicrobial susceptibility testing (AST) for macrolides. We therefore optimized and compared broth microdilution and disk diffusion AST. Multidrug-resistant Gram-negative bacteria (Escherichia coli, Enterobacter cloacae, Klebsiella pneumoniae, Pseudomonas aeruginosa) were tested for azithromycin susceptibility by disk diffusion and broth microdilution in Mueller-Hinton and RPMI-1640 media. Azithromycin susceptibility of Enterobacteriaceae and a subgroup of $P$. aeruginosa increased significantly on RPMI-1640 agar compared to Mueller-Hinton agar. Further, a significant correlation (Kendall, $\tau, p$ ) of zone diameters and minimal inhibitory concentrations (MICs) was found on RPMI-1640 agar for E. coli $(-0.4279,0.0051)$, E. cloacae $(-0.3783,0.0237)$ and P. aeruginosa $(-0.6477$, $<0.0001)$. Performing routine disk diffusion AST on RPMI-1640 agar may lead to the identification of additional therapeutic possibilities for multidrug-resistant bacterial infections in the routine clinical diagnostic setting.
\end{abstract}

Keywords: azithromycin; Gram-negative; Pseudomonas aeruginosa; Enterobacteriaceae; susceptibility testing; MIC; disk diffusion; multidrug-resistant

\section{Introduction}

Increasing bacterial resistance to antibiotics and a lack of new antimicrobial compounds render the treatment of bacterial infections more and more difficult [1]. One example is the rise of pathogens carrying multidrug resistance (MDR) plasmids that confer resistance even against last-resort antibiotics such as carbapenems and colistin. Treatment options for such MDR isolates are extremely limited and the development of new drugs has almost come to a halt over the last two decades [2]. As an alternative approach, old drugs such as fosfomycin or nitrofurantoin, which display a favorable 
epidemiologic susceptibility profile due to infrequent use and concomitant low selection pressure, have been revived. Another approach would be to reconsider susceptibility testing methods for their actual ability to predict the in vivo effectiveness of established drugs against bacterial groups that are currently considered naturally resistant. An example are macrolides which are considered to be active against Gram-positive infections; in clinics they are often used in patients with acute lower respiratory tract infections [3]. Resistances against macrolides in Gram-positive bacteria are often due to methylation or mutations in domain $\mathrm{V}$ of the $23 \mathrm{~S}$ rRNA gene, which is targeted by macrolides, or active efflux [4]. In contrast, most Gram-negative bacteria are considered intrinsically resistant to macrolides, as their outer membrane is highly impermeable to hydrophobic substances and possesses very efficient efflux pump systems [5,6]. While macrolides possess good activity against a few distinct Gram-negative microorganisms, such as Bartonella spp. or Campylobacter spp., they are less effective in Enterobacteriaceae [7,8]. Nevertheless, clinical trials suggest that macrolides could improve the clinical outcome of patients with Gram-negative infections [9,10]. A meta-analysis showed an improved clinical outcome in cystic fibrosis patients routinely treated with macrolides, an effect attributed to immunomodulatory effects [11].

Antimicrobial susceptibility testing (AST) follows Kirby-Bauer's original disk diffusion technique by growing bacteria on cation-adjusted Mueller-Hinton (MH) agar plates, a comparably rich growth medium ensuring sufficient bacterial growth and reproducible results [12]. As azithromycin is not a standard treatment option for Gram-negative infections, susceptibility data and clinical breakpoints have not been published by the European Committee on Antimicrobial Susceptibility Testing (EUCAST) [13]. In contrast with the other macrolides, azithromycin has been shown to be more basic in character, which results in higher permeability across the outer membrane [7]. Thus, recently, a putative minimal inhibitory concentration (MIC) epidemiological cut-off value (ECOFF) of $\geq 32 \mathrm{mg} / \mathrm{L}$ was suggested for Escherichia coli using agar dilution according to the Clinical and Laboratory Standards Institute (CLSI), in order to discriminate wild-type from increased efflux or decreased permeability isolates, and to provide a decision rationale for alternative treatment of diarrheal infections [14].

Bacterial growth media have not been modelled to reflect the physiological situation in the patient but rather to ensure sufficient in vitro growth and reproducibility. Thus, AST in human serum or lung fluids would be ideal but is not feasible in a routine setting. Eukaryotic growth medium has been proposed as an alternative. Recent studies showed that azithromycin MICs were lower in the eukaryotic growth medium Roswell Park Memorial Institute 1640 (RPMI-1640) [15-17] or Dulbecco Modified Eagle Medium (DMEM) as compared to MH medium [18]. Thus, modifying in vitro AST parameters in order to more closely mirror in vivo conditions may reveal additional treatment options for Gram-negative MDR infections [19]. The goal of this study was, hence, to transfer those observations into a routine clinical microbiology setting. As in diagnostics, antibiotic susceptibility is mostly assessed by agar-based disk diffusion testing; we evaluated a quick and simple method for AST by developing a disk diffusion assay on RPMI-1640 agar. This allows to routinely assess the antimicrobial activity of azithromycin against various Gram-negative bacteria.

\section{Results}

\subsection{Enterobacteriaceae Showed Enhanced Azithromycin Susceptibility on RPMI-1640 Agar}

Median azithromycin inhibition zones of Enterobacteriaceae (E. coli, K. pneumoniae and E. cloacae) were significantly larger on RPMI-1640 agar plates as compared to standard MH plates (median diameter increase of 7-8 $\mathrm{mm}$ (Table 1)). 
Table 1. Median azithromycin inhibition zone diameters of Enterobacteriaceae and P. aeruginosa on Mueller-Hinton (MH) and RPMI-1640 agar.

\begin{tabular}{ccccc}
\hline Family & Species & $n$ & MH $[\mathbf{m m}]$ & RPMI-1640 [mm] \\
\hline \multirow{3}{*}{ Enterobacteriaceae } & E. coli & 28 & $15.5(12-17)$ & $24(18.5-26.75)^{* * *}$ \\
\cline { 2 - 5 } & E. cloacae & 24 & $9(6-11)$ & $17.5(15.25-19)^{* * *}$ \\
\cline { 2 - 5 } & K. pneumoniae & 26 & $11.5(9-12.25)$ & $19(16.75-21)^{* * *}$ \\
\hline Pseudomonadaceae & P. aeruginosa & 55 & $6(6-7)$ & $9(7-15)^{* * *}$
\end{tabular}

Azithromycin inhibition zone diameters of Enterobacteriaceae (E. coli, E. cloacae, K. pneumoniae) and P. aeruginosa isolates (26 originating from the University Hospital Zurich (USZ), 29 from four different European study centers) on MH and RPMI-1640 agar. Each isolate was tested in two independent experiments. Data show median + interquartile range. Nonparametric Mann-Whitney Test, ${ }^{* * *}<0.0001$. Disk size: $6 \mathrm{~mm}, 15 \mu \mathrm{g}$ azithromycin. $n=$ number of tested clinical isolates.

The MIC-colored zone method, as described by Kronvall et al., which we adopted and which is used by EUCAST [20], showed significant correlation of MICs and inhibition zone diameters (Figure 1) in RPMI-1640 medium for E. coli and E. cloacae (Kendall's $\tau=-0.4279,-0.3783, p=0.0051$, 0.0237 , respectively). In K. pneumoniae the correlation was not statistically significant although a trend was detectable.
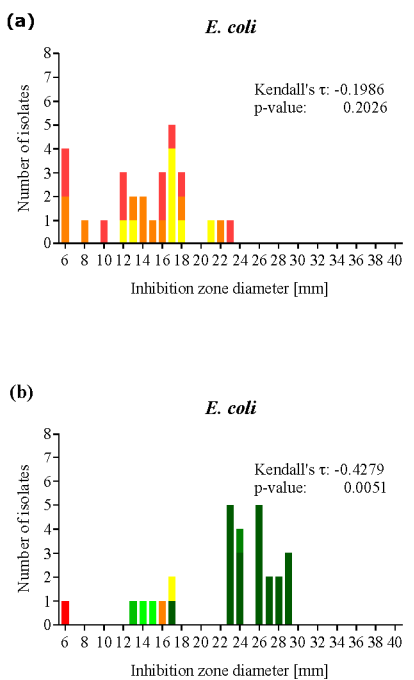
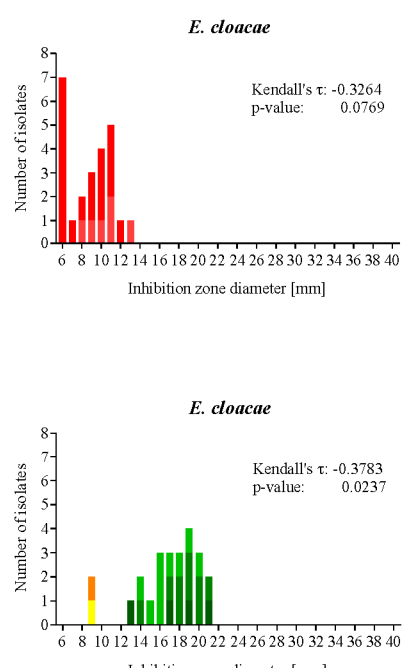

Inhibition zone diameter $[\mathrm{mm}]$
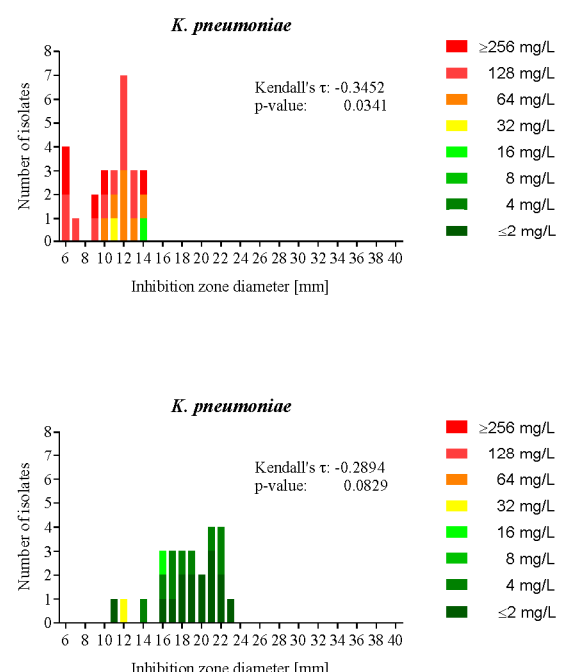

Inhibition zone diameter $[\mathrm{mm}]$

Figure 1. Increased azithromycin susceptibility of Enterobacteriaceae in RPMI-1640 medium as compared to Mueller-Hinton medium. Azithromycin susceptibility of 28 E. coli, 24 E. cloacae and 26 K. pneumoniae isolates in Mueller-Hinton (a); and RPMI-1640 (b); the medium was measured by disk diffusion and broth microdilution. A MIC-colored zone method was applied to correlate the inhibition zone diameter $(\mathrm{mm})$ of each bacterial isolate with the corresponding MIC $(\mathrm{mg} / \mathrm{L})$. Kendall's $\tau$ and p-value were calculated for each species and medium. Each isolate was tested in two independent experiments. Disk size: $6 \mathrm{~mm}, 15 \mu \mathrm{g}$ azithromycin.

The inhibition zone diameter of every individual Enterobacteriaceae isolate increased on RPMI-1640 agar as compared to $\mathrm{MH}$ agar, and for E. coli a putative split into a more susceptible and a resistant population seemed to occur, although corresponding numbers are low (Figure 1). Of note, clinical carbapenemase-positive Enterobacteriaceae strains consistently showed larger azithromycin inhibition zones on RPMI-1640 than on MH agar (Figure S1). Inhibition zones for the other antibiotics tested (cefepime, tobramycin, clarithromycin, erythromycin, tetracycline and tigecycline) did not differ between RPMI-1640 and MH agar in any of the examined species. In contrast to our freshly prepared RPMI-1640 agar plates, the commercially available RPMI-1640 agar plates routinely used for mycology assays were not suitable due to inhibition of bacterial growth. To exclude the possibility that the 
increased susceptibility found in RPMI-1640 was exclusively due to the added sodium bicarbonate, we assessed the azithromycin MICs in MH with sodium bicarbonate in a set of four strains of each species. Consistent with the data of Ersoy et al. [18], the MICs towards azithromycin were lower in MH with sodium bicarbonate compared to $\mathrm{MH}$ without sodium bicarbonate. However, the tested isolates remained less susceptible to azithromycin in MH with sodium bicarbonate as compared to RPMI-1640 (Table S2). Thus, we conclude that sodium bicarbonate is not the only component in RPMI-1640 leading to lower resistance towards azithromycin.

\subsection{Azithromycin Susceptibility Increased on RPMI-1640 Agar in a Subgroup of P. aeruginosa}

In the 55 clinical P. aeruginosa isolates, the average azithromycin inhibition zone diameters increased significantly on RPMI-1640 agar as compared to MH agar, although to a lower extent than in the Enterobactericeae strains (Table 1). This was mainly due to a subgroup of strains displaying inhibition zone diameters ranging from 6 to $12 \mathrm{~mm}$ with minimal difference between RPMI-1640 and $\mathrm{MH}$ agar. In contrast, two other delimited sub-populations showed a trend towards higher azithromycin susceptibility on RPMI-1640 as compared to MH agar, one group ranging from 13 to $20 \mathrm{~mm}$ and the other group displaying large inhibition zone diameters (24 to $30 \mathrm{~mm}$ ) on RPMI-1640 agar (Figure 2).

(a)

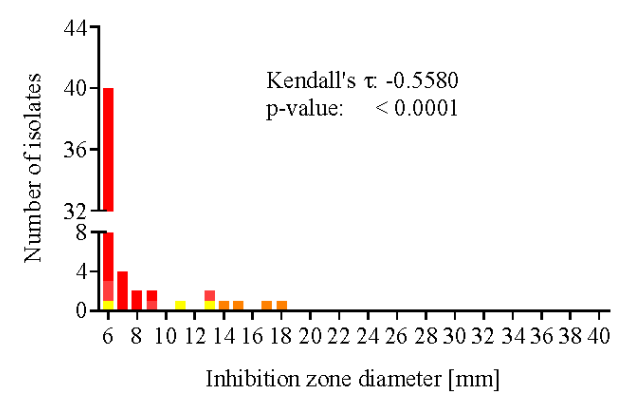

(b)

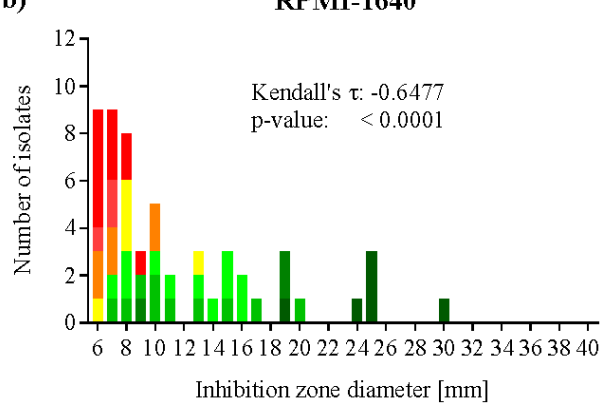

$\geq 256 \mathrm{mg} / \mathrm{L}$

- $128 \mathrm{mg} / \mathrm{L}$

- $\quad 64 \mathrm{mg} / \mathrm{L}$

$32 \mathrm{mg} / \mathrm{L}$

$16 \mathrm{mg} / \mathrm{L}$

$8 \mathrm{mg} / \mathrm{L}$

- $4 \mathrm{mg} / \mathrm{L}$

- $\leq 2 \mathrm{mg} / \mathrm{L}$

Figure 2. Increased azithromycin susceptibility in a subgroup of $P$. aeruginosa in RPMI-1640 medium as compared to Mueller-Hinton medium. Azithromycin susceptibility of 55 P. aeruginosa isolates in Mueller-Hinton (a); and RPMI-1640 (b); the medium was measured by disk diffusion and broth microdilution. MIC-zone method was applied to correlate the inhibition zone diameter [mm] of each bacterial isolate with the corresponding MIC (mg/L). Kendall's $\tau$ and p-value were calculated for each species and medium. Each isolate was tested in two independent experiments. Disk size: $6 \mathrm{~mm}$, $15 \mu \mathrm{g}$ azithromycin.

Nineteen out of the 29 clinical P. aeruginosa isolates from the European study batch derived from cystic fibrosis patients. Ten of those isolates harbored a mutation in the gene coding for Domain $\mathrm{V}$ of the $23 \mathrm{~S}$ ribosomal subunit, which is known to confer azithromycin resistance [21]. All the isolates containing a mutation presented with small inhibition zone diameters ranging from 6-8 mm and MICs $\geq 128 \mathrm{mg} / \mathrm{L}$ in both media, while the rest of the CF patients' isolates showed increased susceptibility in RPMI-1640 medium (Figure 3). None of the hospital-acquired isolates $(n=10)$ carried a mutation. Testing the 26 clinical isolates collected at the University Hospital of Zurich revealed that only one strain harbored a mutation (A2045T), which corresponded with an azithromycin-resistant phenotype on both media measured by disk diffusion, as well as by broth microdilution. Despite displaying a wild-type $23 \mathrm{~S}$ rRNA gene, 15 out of 44 isolates presented with a resistant profile on MH and RPMI-1640 agar but showed lower MICs on RPMI-1640 than on MH medium (Figure 3). 
(a)

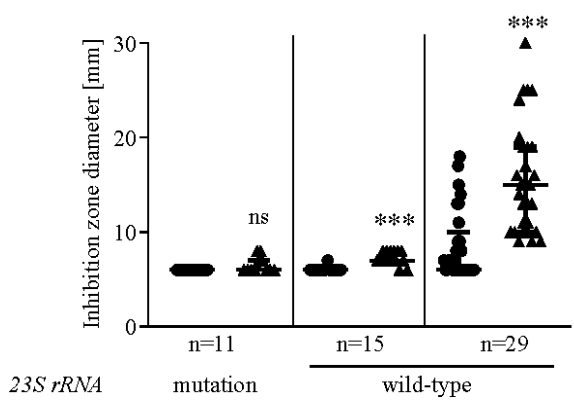

(b)

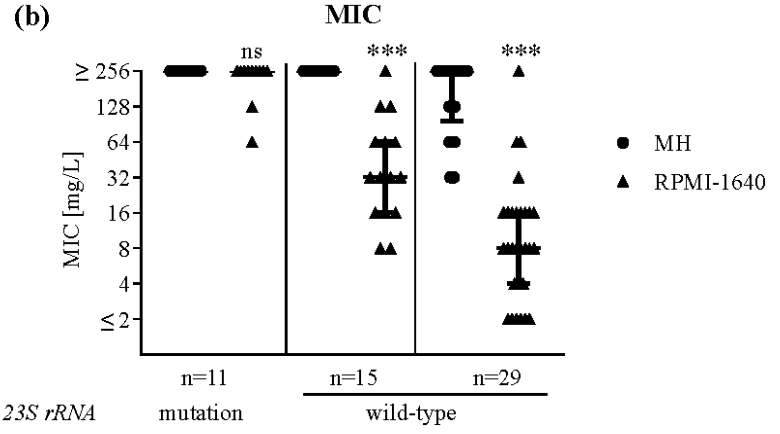

Figure 3. P. aeruginosa isolates carrying a mutation in Domain $\mathrm{V}$ of the $23 S \mathrm{r} R A$ gene showed a resistant profile in liquid and solid Mueller-Hinton and RPMI-1640 medium. Domain V of the $23 S$ rRNA gene was sequenced in 55 P. aeruginosa isolates and analyzed for known mutations conferring resistance to macrolides (A2045T, A2045G, A2046T, A2046G and C2598T). Isolates with a wild-type Domain V of the $23 S$ rRNA gene were split into two subgroups according to their resistance profile on RPMI-1640 agar. Azithromycin susceptibility was measured by disk diffusion (a); and broth microdilution (b); in Mueller-Hinton (circles) and RPMI-1640 (triangles) medium. Data show the median + interquartile range, Nonparametric Mann-Whitney Test, ${ }^{* * *}<0.0001$. Disk size: $6 \mathrm{~mm}, 15 \mu \mathrm{g}$ azithromycin.

\section{Discussion}

In this study, we showed that the azithromycin susceptibility of clinical Enterobacteriaceae isolates was higher on RPMI-1640 agar than on the routinely used MH agar. These findings take the previous observations, showing lower MICs in liquid tissue culture media (DMEM and RPMI-1640) than in MH broth for several bacterial species, e.g., Stenotrophomonas, a step further, setting the stage to expand routine AST testing in the clinical microbiology laboratory [16-18,21]. To the best of our knowledge, this is the first method comparison study comprising the most relevant Gram-negative bacterial species regarding antibiotic susceptibility on MH and RPMI solid and liquid media. The assumption that the growth medium has no influence on the predictive power of susceptibility testing for therapeutic effectivity is therefore put into question; and careful evaluation and revision of in vitro AST methods might be necessary for specific situations, such as infections with multidrug-resistant Gram-negative bacteria.

The observed differences in susceptibility suggest the existence of determinants influencing antibiotic susceptibility that may be active in the nutrient-rich $\mathrm{MH}$ agar and inactive in the more physiological and nutrient-poor RPMI-1640 agar. Ersoy et al. previously showed that the increased susceptibility of azithromycin, erythromycin and tetracycline in liquid DMEM was influenced by the presence of sodium bicarbonate in DMEM [18]. Although RPMI-1640 contains similar concentrations of sodium bicarbonate as DMEM, only the susceptibility towards azithromycin was affected in the disk diffusion assay in our study (Table S3).

As a possible mechanism for the increased azithromycin susceptibility of $P$. aeruginosa in RPMI-1640 medium, Buyck et al. proposed an increase of the outer membrane permeability. Thereby, the macrolides are able to penetrate the membrane and, through a yet unknown mechanism, impair the expression of oprM, the outer membrane component of one of the main efflux systems $[17,22,23]$. The distinct susceptibility phenotypes we observed amongst the tested P. aeruginosa strains (Figure 2; Figure 3) suggest that RPMI-1640 medium induces additionally a specific response in distinct bacterial populations. Such a response might be caused by point mutations in the gene coding for Domain $\mathrm{V}$ of the $23 \mathrm{~S}$ rRNA, which are known to increase resistance towards azithromycin in P. aeruginosa [24]. Mustafa et al. analyzed a collection of over 300 clinical P. aeruginosa isolates for $23 S$ rRNA mutations and discovered single or double mutations in $43 \%$ of the isolates with cystic fibrosis origin [21]. In contrast, no mutations were found in isolates from patients with hospital-acquired pneumonia. In our P. aeruginosa collection, where cystic fibrosis patients were a minority, we found only one isolate carrying a point mutation in the $23 S \mathrm{rRNA}$ gene. Nevertheless, additional 15 isolates without mutation 
showed similar inhibition zone diameters on both media but lower MICs in RPMI-1640 medium. While resistance in a liquid medium correlated with the known mutations in Domain V of the 23S rRNA gene, these mutations seemed to not be the sole determinants of decreased susceptibility on RPMI-1640 agar. Therefore, we assume that yet another mechanism is responsible for the medium-dependent shift in susceptibility measured by disk diffusion in our clinical isolate collection.

In a murine infection model of MDR Gram-negative bacterial infections, azithromycin was found to improve disease outcome [15,25]. Further, azithromycin was shown to reduce absolute bacterial numbers and increase specialized metabolite production of P. aeruginosa biofilms [26]. Thus, not only immunomodulatory effects but also impairment of planktonic and biofilm-associated bacteria may play an important role for the beneficial effects of macrolide therapy found in patients with Gram-negative sepsis or ventilator-associated pneumonia [9,11,26-29].

While several studies showed increased azithromycin MICs in tissue culture media as compared to $\mathrm{MH}$ in various Gram-negative species, AST by disk diffusion on tissue culture medium has not been tested so far. Comparison of the two methods revealed excellent MIC/zone correlations on RPMI-1640 agar in E. coli, E. cloacae and P. aeruginosa, a trend was observed in K. pneumoniae. These findings could pave the way for further expanding the well-established, accurate, simple, fast, and reproducible disk diffusion method from MH agar to solid RPMI-1640 to the clinical microbiology routine testing. This may broaden the potential therapeutic options in multidrug-resistant bacterial infections, eliminating major errors in forecasting therapeutic success in susceptibility reports.

In summary, we have demonstrated that the inhibition zone diameters correlated well with the MICs in both MH as well as RPMI-1640 medium. Azithromycin inhibition zone diameters significantly increased on RPMI-1640 agar when tested with the disk diffusion method, both for fully susceptible as well as multidrug-resistant Gram-negatives, including carbapenemase-producing Enterobacteriaceae. We took previous findings a step further by rendering them applicable in the routine setting of a clinical microbiology laboratory. Thus, we propose that for multidrug-resistant Gram-negative bacteria additional testing conditions, which more closely reflect the host environment, e.g., cultivation on RPMI-1640 agar, should be considered in routine testing in defined situations, such as assessing additional therapeutic options for multidrug-resistant Gram-negative bacteria. This may unveil additional susceptibilities to active antimicrobial substances and pave the way to clinical studies correlating standardized in vitro AST data with therapeutic outcome in vivo in order to further assess the therapeutic utility of macrolides in Gram-negative infections.

\section{Materials and Methods}

\subsection{Bacterial Strains and Media}

The azithromycin susceptibility of 28 Escherichia coli, 24 Enterobacter cloacae, 26 Klebsiella pneumoniae and 26 Pseudomonas aeruginosa isolates, collected between 2014 and 2016 at the University Hospital Zurich (USZ), was tested by disk diffusion and broth microdilution techniques. Carbapenemase-positive isolates were collected between 2013 and 2015 (14 K. pneumoniae, 2 E. coli, 1 Proteus mirabilis, 1 Citrobacter koseri, 1 Morganella morganii) and tested by disk diffusion. Additionally, 29 clinical P. aeruginosa isolates, which were collected in 4 different European study centers and had previously been tested for their susceptibility to macrolides including azithromycin by broth microdilution, [21] were now assessed by disk diffusion. Strains were stored at $-80{ }^{\circ} \mathrm{C}$ in $\mathrm{LB} / 20 \%$ glycerol and grown in Luria Broth Lennox (LB, Becton Dickinson, Le Pont de Claix, France) or on cation-adjusted Mueller-Hinton II (MH, BD, France) or Roswell Park Memorial Institute agar (RPMI-1640, Gibco, Paisley, UK) in a shaking incubator at $35{ }^{\circ} \mathrm{C}, 5 \% \mathrm{CO}_{2}$. RPMI-1640 was prepared according to the manufacturer's instructions (including the addition of $2 \mathrm{~g} / \mathrm{L} \mathrm{NaHCO}_{3}$ ), filter-sterilized, and the $\mathrm{pH}$ was adjusted to 7.0-7.4. RPMI-1640 agar plates consisted of 72\% RPMI-1640, 1\% agar (BD, France) and 3\% LB. RPMI-1640 agar plates (Biomérieux, Marcy-L'Etoile, France) were used for comparison. 


\subsection{Disk Diffusion Testing}

Disk diffusion testing was performed according to EUCAST guidelines using commercial MH agar square plates (Becton Dickinson, Franklin Lakes, NJ, USA) and custom-made RPMI-1640 agar plates (as described above) [30]. After placing antibiotic paper disks (azithromycin, erythromycin, clarithromycin, tobramycin, cefepime, tetracycline, tigecycline, i2a, France; for concentrations see Table S1), plates were incubated ( 18 hours, $35^{\circ} \mathrm{C}$ ) with $5 \% \mathrm{CO}_{2}$ to maintain a stable plate $\mathrm{pH}$ since RPMI-1640 was buffered with sodium bicarbonate. Inhibition zones were detected by the SirScan 2000 automatic plate reader (i2a). Two independent measurements were performed on each isolate. The quality control protocol (QC), as recommended by EUCAST for MH agar, was routinely followed also for RPMI-1640 agar to ensure standardization of the technique and quality of the agar plates [31]. Inhibition zones of the two EUCAST reference strains P. aeruginosa ATCC 27853 and E. coli ATCC 25922 matched EUCAST QC requirements on $\mathrm{MH}$ agar plates, confirming constant quality of our testing procedure.

\subsection{Broth Microdilution Susceptibility Testing}

The minimal inhibitory concentration (MIC) of azithromycin in liquid MH or RPMI-1640 medium was assessed by broth microdilution following the EUCAST protocol [32]. Differing from the EUCAST protocol, the plates were incubated for 20 hours at $37^{\circ} \mathrm{C}$ in a shaking incubator supplemented with $5 \%$ $\mathrm{CO}_{2}$ to keep the $\mathrm{pH}$ of the RPMI-1640 medium stable. Assays were performed in duplicates.

\subsection{Sequencing of the Gene Coding for Domain V of the $23 S$ Ribosomal Subunit}

Domain $\mathrm{V}$ of the $23 S \mathrm{R} N A$ gene was amplified and sequenced as described before and screened for the previously described mutations A2045G/T, A2046G/T and C2598G/T in P. aeruginosa strains [21].

\subsection{Statistical Analysis}

The statistical analysis was performed with either GraphPad Prism 7.04 (Mann-Whitney Test) (GraphPad Software, San Diego, CA, USA) or R version 3.5.0 (Kendall rank coefficient) (GraphPad Software, San Diego, CA, USA) software for Windows.

Supplementary Materials: The following are available online at http://www.mdpi.com/2079-6382/9/5/218/s1, Figure S1: Increased azithromycin susceptibility in carbapenemase-positive Enterobacteriaceae on RPMI-1640 agar as compared to MH agar. Table S1: Concentration of antibiotic disks. Table S2: Decrease in resistance towards azithromycin in RPMI-1640 or MH containing $\mathrm{NaHCO}_{3}$. Table S3: Median erythromycin and tetracycline inhibition zone diameters of Enterobacteriaceae and P. aeruginosa on MH and RPMI-1640 agar.

Author Contributions: Conceptualization, A.S.Z., M.M. and A.T.; formal analysis M.M., A.T., M.B., M.H. and A.S.Z.; investigation: M.M., A.T. and M.B.; methodology, A.S.Z., M.H., M.M. and A.T.; writing-original draft preparation, M.M., A.T., A.S.Z. and M.H.; writing-review and editing, A.S.Z., M.H., and F.V.B.; funding acquisition, A.S.Z. All authors have read and agreed to the published version of the manuscript.

Funding: This research was funded by the Swiss National Foundation (grant number 310030_146295/1) to ASZ. FVB is Research Director of the Belgian Fonds National de la Recherche Scientifique.

Acknowledgments: We thank Reinhard Zbinden and the technicians of the Institute of medical microbiology of the University of Zürich for expert help and assistance, Federica Andreoni and Reto Schüpbach for ideas and discussions, Roger Kouyos and Bruno Ledergerber for help with statistics.

Conflicts of Interest: The authors declare no conflict of interest. The funders had no role in the design of the study; in the collection, analyses, or interpretation of data; in the writing of the manuscript, or in the decision to publish the results.

\section{References}

1. Deak, D.; Outterson, K.; Powers, J.H.; Kesselheim, A.S. Progress in the Fight against Multidrug-Resistant Bacteria? A Review of U.S. Food and Drug Administration-Approved Antibiotics, 2010-2015. Ann. Intern. Med. 2016, 165, 363. [CrossRef]

2. Outterson, K.; Powers, J.H.; Daniel, G.W.; McClellan, M.B. Repairing the Broken Market for Antibiotic Innovation. Health Aff. 2015, 34, 277-285. [CrossRef] 
3. Laopaiboon, M.; Panpanich, R.; Mya, K.S. Azithromycin for acute lower respiratory tract infections. Cochrane Database Syst. Rev. 2015, 2015, CD001954. [CrossRef]

4. Leclercq, R. Mechanisms of Resistance to Macrolides and Lincosamides: Nature of the Resistance Elements and Their Clinical Implications. Clin. Infect. Dis. 2002, 34, 482-492. [CrossRef]

5. Vaara, M. Outer membrane permeability barrier to azithromycin, clarithromycin, and roxithromycin in gram-negative enteric bacteria. Antimicrob. Agents Chemother. 1993, 37, 354-356. [CrossRef]

6. Schweizer, H.P. Efflux as a mechanism of resistance to antimicrobials in Pseudomonas aeruginosa and related bacteria: Unanswered questions. Genet. Mol. Res. 2003, 2, 48-62. [PubMed]

7. Gomes, C.; Martínez-Puchol, S.; Palma, N.; Horna, G.; Ruiz-Roldán, L.; Pons, M.J.; Ruiz, J. Macrolide resistance mechanisms in Enterobacteriaceae: Focus on azithromycin. Crit. Rev. Microbiol. 2016, 43, 1-30. [CrossRef]

8. Lübbert, C. Antimicrobial therapy of acute diarrhoea: A clinical review. Expert Rev. Anti-infective Ther. 2015, 14, 193-206. [CrossRef] [PubMed]

9. Giamarellos-Bourboulis, E.J.; Mylona, V.; Antonopoulou, A.; Tsangaris, I.; Koutelidakis, I.; Marioli, A.; Raftogiannis, M.; Kopterides, P.; Lymberopoulou, K.; Mouktaroudi, M.; et al. Effect of clarithromycin in patients with suspected Gram-negative sepsis: Results of a randomized controlled trial. J. Antimicrob. Chemother. 2013, 69, 1111-1118. [CrossRef] [PubMed]

10. Giamarellos-Bourboulis, E.J.; Pechère, J.-C.; Routsi, C.; Plachouras, D.; Kollias, S.; Raftogiannis, M.; Zervakis, D.; Baziaka, F.; Koronaios, A.; Antonopoulou, A.; et al. Effect of Clarithromycin in Patients with Sepsis and Ventilator-Associated Pneumonia. Clin. Infect. Dis. 2008, 46, 1157-1164. [CrossRef] [PubMed]

11. Cai, Y.; Chai, D.; Wang, R.; Bai, N.; Liang, B.-B.; Liu, Y. Effectiveness and safety of macrolides in cystic fibrosis patients: A meta-analysis and systematic review. J. Antimicrob. Chemother. 2011, 66, 968-978. [CrossRef] [PubMed]

12. Bauer, A.W.; Kirby, W.M.M.; Sherris, J.C.; Turck, M. Antibiotic Susceptibility Testing by a Standardized Single Disk Method. Am. J. Clin. Pathol. 1966, 45, 493-496. [CrossRef] [PubMed]

13. European Committee on Antimicrobial Susceptibility Testing. Breakpoint Tables for Interpretation of MICs and Zone Diameters. Version 8.1. 2018. Available online: https://www.eucast.org/ast_of_bacteria/previous_ versions_of_documents/ (accessed on 28 April 2020).

14. Gomes, C.; Ruiz-Roldán, L.; Mateu, J.; Ochoa, T.J.; Ruiz, J. Azithromycin resistance levels and mechanisms in Escherichia coli. Sci. Rep. 2019, 9, 6089. [CrossRef] [PubMed]

15. Lin, L.; Nonejuie, P.; Munguia, J.; Hollands, A.; Olson, J.; Dam, Q.; Kumaraswamy, M.; Rivera, H.; Corriden, R.; Rohde, M.; et al. Azithromycin Synergizes with Cationic Antimicrobial Peptides to Exert Bactericidal and Therapeutic Activity Against Highly Multidrug-Resistant Gram-Negative Bacterial Pathogens. EBioMedicine 2015, 2, 690-698. [CrossRef]

16. Kumaraswamy, M.; Lin, L.; Olson, J.; Sun, C.-F.; Nonejuie, P.; Corriden, R.; Döhrmann, S.; Ali, S.R.; Amaro, D.; Rohde, M.; et al. Standard susceptibility testing overlooks potent azithromycin activity and cationic peptide synergy against MDR Stenotrophomonas maltophilia. J. Antimicrob. Chemother. 2016, 71, 1264-1269. [CrossRef]

17. Buyck, J.M.; Plesiat, P.; Traore, H.; Vanderbist, F.; Tulkens, P.M.; Van Bambeke, F. Increased Susceptibility of Pseudomonas aeruginosa to Macrolides and Ketolides in Eukaryotic Cell Culture Media and Biological Fluids Due to Decreased Expression of oprM and Increased Outer-Membrane Permeability. Clin. Infect. Dis. 2012, 55, 534-542. [CrossRef]

18. Ersoy, S.C.; Heithoff, D.M.; Barnes, L.; Tripp, G.K.; House, J.K.; Marth, J.D.; Smith, J.W.; Mahan, M.J. Correcting a Fundamental Flaw in the Paradigm for Antimicrobial Susceptibility Testing. EBioMedicine 2017, 20, 173-181. [CrossRef]

19. Hirsch, E.B.; Tam, V.H. Detection and treatment options for Klebsiella pneumoniae carbapenemases (KPCs): An emerging cause of multidrug-resistant infection. J. Antimicrob. Chemother. 2010, 65, 1119-1125. [CrossRef]

20. Kronvall, G.; Giske, C.G.; Kahlmeter, G. Setting interpretive breakpoints for antimicrobial susceptibility testing using disk diffusion. Int. J. Antimicrob. Agents 2011, 38, 281-290. [CrossRef]

21. Mustafa, M.-H.; Khandekar, S.; Tunney, M.; Elborn, J.S.; Kahl, B.C.; Denis, O.; Plésiat, P.; Traore, H.; Tulkens, P.M.; Vanderbist, F.; et al. Acquired resistance to macrolides in Pseudomonas aeruginosa from cystic fibrosis patients. Eur. Respir. J. 2017, 49, 1601847. [CrossRef] 
22. Li, X.Z.; Nikaido, H.; Poole, K. Role of mexA-mexB-oprM in antibiotic efflux in Pseudomonas aeruginosa. Antimicrob. Agents Chemother. 1995, 39, 1948-1953. [CrossRef] [PubMed]

23. Masuda, N.; Sakagawa, E.; Ohya, S.; Gotoh, N.; Tsujimoto, H.; Nishino, T. Substrate Specificities of MexAB-OprM, MexCD-OprJ, and MexXY-OprM Efflux Pumps in Pseudomonas aeruginosa. Antimicrob. Agents Chemother. 2000, 44, 3322-3327. [CrossRef] [PubMed]

24. Marvig, R.L.; Søndergaard, M.S.R.; Damkiær, S.; Høiby, N.; Johansen, H.K.; Molin, S.; Jelsbak, L. Mutations in $23 \mathrm{~S}$ rRNA Confer Resistance against Azithromycin in Pseudomonas aeruginosa. Antimicrob. Agents Chemother. 2012, 56, 4519-4521. [CrossRef] [PubMed]

25. Retsema, J.A.; Girard, A.E.; Milisen, W.B. Relationship of high tissue concentrations of azithromycin to bactericidal activity and efficacy in vivo. J. Antimicrob. Chemother. 1990, 25, 83-89. [CrossRef] [PubMed]

26. Phelan, V.; Fang, J.; Dorrestein, P.C. Mass Spectrometry Analysis of Pseudomonas aeruginosa Treated with Azithromycin. J. Am. Soc. Mass Spectrom. 2015, 26, 873-877. [CrossRef]

27. Wolter, J.; Seeney, S.; Bell, S.C.; Bowler, S.; Masel, P.; McCormack, J. Effect of long term treatment with azithromycin on disease parameters in cystic fibrosis: A randomised trial. Thorax 2002, 57, 212-216. [CrossRef]

28. Kanoh, S.; Rubin, B.K. Mechanisms of Action and Clinical Application of Macrolides as Immunomodulatory Medications. Clin. Microbiol. Rev. 2010, 23, 590-615. [CrossRef]

29. Saini, H.; Chhibber, S.; Harjai, K. Azithromycin and ciprofloxacin: A possible synergistic combination against Pseudomonas aeruginosa biofilm-associated urinary tract infections. Int. J. Antimicrob. Agents 2015, 45, 359-367. [CrossRef]

30. Matuschek, E.; Brown, D.; Kahlmeter, G. Development of the EUCAST disk diffusion antimicrobial susceptibility testing method and its implementation in routine microbiology laboratories. Clin. Microbiol. Infect. 2014, 20, O255-O266. [CrossRef]

31. The European Committee on Antimicrobial Susceptibility Testing. Routine and Extended Internal Quality Control for MIC Determination and Disk Diffusion as Recommended by EUCAST. Version 6.1. 2016. Available online: https://www.eucast.org/ast_of_bacteria/previous_versions_of_documents/ (accessed on 28 April 2020).

32. European Committee on Antimicrobial Susceptibility Testing. Determination of minimum inhibitory concentrations (MICs) of antibacterial agents by broth dilution. Clin. Microbiol. Infect. 2003, 9, 9-15. [CrossRef]

(C) 2020 by the authors. Licensee MDPI, Basel, Switzerland. This article is an open access article distributed under the terms and conditions of the Creative Commons Attribution (CC BY) license (http://creativecommons.org/licenses/by/4.0/). 